\title{
Repeatability and Relationship of Incidence and Severity Measures of Scab of Wheat Caused by Fusarium graminearum in Inoculated Nurseries
}

\author{
J. V. Groth and E. A. Ozmon, Department of Plant Pathology, and R. H. Busch, Department of Agronomy and \\ Plant Genetics, University of Minnesota, St. Paul 55108
}

\begin{abstract}
Groth, J. V., Ozmon, E. A., and Busch, R. H. 1999. Repeatability and relationship of incidence and severity measures of scab of wheat caused by Fusarium graminearum in inoculated nurseries. Plant Dis. 83:1033-1038.

Breeders of hard red spring wheat (Triticum aestivum) are attempting to incorporate resistance to scab, caused by Fusarium graminearum. In artificially inoculated, replicated field plots, 37 wheat entries (inbred lines or cultivars) were evaluated for 3 years and an additional 60 entries for 2 of the 3 years for incidence (percent spikes infected), severity (percent infected spikelets within infected spikes), and disease index (percent infected spikelets in 50-spike sample). From year to year, entries had similar index values, with coefficients of determination $\left(r^{2}\right)$ ranging from 0.59 to 0.78 , with a mean of 0.73 . Entries appeared slightly more similar from year to year for incidence than for severity, although both measures of disease had highly significant $r^{2}$ values. Incidence and severity were highly correlated in the wheat germ plasm examined; $r^{2}$ values in single years ranged from 0.51 to 0.67 , with a mean of 0.64 . A representative subset of 22 entries was included for a fourth year. None of the measures of disease in year 4 correlated with their counterparts in any of the first 3 years. This loss of repeatability may have been caused by severe lodging or by high temperatures during the evaluation period that accelerated disease progress and wheat maturity during year 4 . Incidence and severity remained correlated in year 4 $\left(r^{2}=0.60\right)$.
\end{abstract}

Additional keywords: components of resistance, genotype $\times$ environment interaction, head blight

Scab, caused by Fusarium graminearum, is a serious disease of wheat (Triticum aestivum) and barley (Hordeum vulgare) in more humid growing areas of the world (14). At present, the only effective controls for this disease are clean tillage and host resistance. Elite wheats in the United States are moderately to fully susceptible. Certain Chinese wheat cultivars have been developed with high degrees of scab resistance, based on a small but unknown number of resistant sources (1). The resistances of Chinese cultivars have been or are currently being incorporated into elite wheat lines in several wheat breeding programs in the United States and Canada. Other sources of high-level resistance are being sought, and currently available elite wheat lines and cultivars (hereafter referred to simply as "lines") are being evaluated for lower degrees of resistance for immediate use.

Methods have been described for inoculum production, inoculation of field nurseries, and irrigation of nurseries to encourage epidemics of scab $(4,15)$. Using

Corresponding author: J. V. Groth

E-mail: jamesg@puccini.crl.umn.edu

Accepted for publication 2 August 1999.

Publication no. D-1999-0909-01R

(C) 1999 The American Phytopathological Society these methods, we have maintained inoculated scab screening nurseries at St. Paul, Minnesota, every year since 1985 . These nurseries allow screening of germ plasm in the field, where both incidence (proportion of infected spikes to total spikes) and severity (proportion of infected spikelets on infected spikes) can be measured. Data from these inoculated plots now can provide an assessment on reliability of field evaluation of scab resistance and the relationship between incidence and severity in wheat germ plasm.

Our objectives in this 3-year study were (i) to examine year-to-year repeatability of the incidence and severity scab measurements, both relatively and absolutely, (ii) to determine the relationship between these measures, since incidence and severity measure two putatively distinct components of scab resistance, and (iii) to determine whether components of resistance could be identified and possibly combined from separate sources to provide higher levels of resistance.

\section{MATERIALS AND METHODS}

Selection of wheat germ plasm. Nurseries used in this study are part of a large public USDA breeding program for hard red spring wheats at the University of Minnesota. Disease evaluations were made in 1992 through 1995 in these breeding nurseries at St. Paul. Germ plasm was selected for inclusion because it was (i) advanced material with desirable agronomic characteristics, (ii) early generation material, including exotic putative sources of resistance, for initial evaluation, or (iii) popular current or historic cultivars with known yield and quality characteristics. Breeding lines were at the $F_{8}$ to $F_{10}$ (or higher in later years) generations, and were advanced as pure lines by taking seeds from several heads. Generally, most lines were not included solely on the basis of scab resistance. The nurseries included lines of all degrees of scab susceptibility. In the later stages of the study, many lines included in the first or second year were eliminated or modified as material in the breeding program was advanced.

Each line was replicated three times as single 3 - or $5-\mathrm{m}$ rows with 60 to 100 plants per row. A randomized complete block design was used for each of the several trials included each year.

Inoculum. Fresh isolates of $F$. graminearum were obtained from diseased seeds from five different areas of Minnesota using isolation, culturing, and identification techniques described by Nelson et al. (10). On average, 15 isolates from each area were used. In 1992, all inoculum was increased on potato dextrose agar (PDA). In 1993, corn meal agar (40 $\mathrm{g}$ of corn meal, 1 liter of distilled water, and $15 \mathrm{~g}$ of Bacto agar) was substituted for PDA for about half of the increase, and in 1994, mung bean agar (40 g of dry mung beans boiled for $1 \mathrm{~h}, 1$ liter of distilled water, and $15 \mathrm{~g}$ of Bacto agar) and PDA were used about equally to increase the inoculum. Corn meal and mung bean agar were used because they allow for greater spore production and permit a shorter (1- to 2-week) growth period compared with 2 to 4 weeks typically required for cultures on PDA. All media were prepared from fresh ingredients. Five different isolates were placed on each $9-\mathrm{cm}$ plate. The contents of 25 plates were placed in $600 \mathrm{ml}$ of deionized water in a blender and blended on high. The volume was brought up to 1.25 liters and blended again for $1 \mathrm{~min}$. The suspension was filtered through several layers of cheesecloth. Spore counts of each batch were made with a hemacytometer. Counts averaged $1.6 \times 10^{4}$ spores per $\mathrm{ml}$ in 1992 , $1.4 \times 10^{5}$ in $1993,1.7 \times 10^{5}$ in 1994 , and $3.9 \times 10^{4}$ in 1995 . Mycelial fragments outnumbered spores several-fold, but no attempt was made to count them. 
Inoculation. Each line in the nursery was inoculated beginning at the time of emergence of the main spike from the flag leaf sheath. Inoculation was done on alternate evenings, and each line received at least six inoculations. About 25 liters of inoculum was used to cover the entire nursery after it was diluted 1:10 with water. Dilute inoculum was applied to the plots just above plant height with a doubleboom tractor-drawn sprayer at $2.1 \mathrm{~kg} / \mathrm{cm}^{2}$ pressure at the nozzles, at a rate of 935 liters/ha. Booms were $3 \mathrm{~m}$ long, with cone spray nozzles at $0.3-\mathrm{m}$ intervals $1.3 \mathrm{~m}$ above the ground. Inoculated plants were misted with overhead irrigation for several minutes after inoculation and two to three times during the daylight hours (15).

Disease assessment. Disease intensity was evaluated as late in plant maturity as possible, but while glumes were still green. growth varied with the year: in 1992, it occurred 36 days after spike emergence for The time required to reach this stage of

most lines; whereas in 1993, 1994, and 1995, it occurred at 27, 24, and 19 days, respectively. Fifty arbitrarily selected, dominant spikes (main culms) in each row were scored to record both incidence (number of heads with necrotic scab symptoms) and severity (number of necrotic spikelets in each infected spike divided by number of spikelets in infected spikes). Scoring severity only on infected spikes removes autocorrelation with incidence that might result if severity were calculated from both infected and noninfected spikes. A disease index was calculated as the number of infected spikelets over total spikelets in 50 heads, and is equivalent to the product of incidence and severity.

Statistical analysis. Means of the three replicates for incidence, severity, and disease index were calculated for each entry each year, and all tables and figures are based on these means. Both correlation analysis and regression analysis were used

Table 1. Ranked mean scab index (percentage of diseased spikelets), scab incidence (percentage of diseased spikes), and sources for 37 spring wheat lines included in inoculated, replicated field plots at St. Paul, Minnesota, in 1992 to 1994

\begin{tabular}{|c|c|c|c|}
\hline Line & $\begin{array}{l}\text { 3-year mean } \\
\text { index }\end{array}$ & $\begin{array}{c}\text { 3-year mean } \\
\text { incidence }\end{array}$ & Source \\
\hline Sumai $3^{\mathrm{a}}$ & 0.19 & 2.73 & China \\
\hline Selections $1-13^{\mathrm{a}, \mathrm{b}}$ & $1.05-2.54$ & $12.01-25.55$ & Pioneer Hi-bred \\
\hline Butte $86^{a}$ & 2.94 & 22.77 & North Dakota State University \\
\hline Sharp ${ }^{\mathrm{a}}$ & 3.12 & 27.32 & South Dakota State University \\
\hline $2375^{\mathrm{a}}$ & 4.54 & 34.22 & Pioneer Hi-bred \\
\hline Trenton & 4.69 & 35.22 & North Dakota State University \\
\hline Stoa $^{\mathrm{a}}$ & 6.64 & 39.78 & North Dakota State University \\
\hline $2370^{\mathrm{a}}$ & 6.89 & 50.21 & Pioneer Hi-bred \\
\hline Kulm & 6.94 & 51.34 & North Dakota State University \\
\hline Russ & 7.15 & 42.21 & South Dakota State University \\
\hline Verde & 8.05 & 49.77 & University of Minnesota \\
\hline Grandin ${ }^{a}$ & 8.08 & 50.78 & North Dakota State University \\
\hline Marshalla $^{\mathrm{a}}$ & 8.90 & 53.55 & University of Minnesota \\
\hline Nordic $^{\mathrm{a}}$ & 10.25 & 61.77 & AgriPro \\
\hline Prospect $^{\mathrm{a}}$ & 10.44 & 54.00 & South Dakota State University \\
\hline Bergen $^{a}$ & 11.08 & 55.57 & AgriPro \\
\hline 2371 & 11.84 & 65.78 & Pioneer Hi-bred \\
\hline Gus $^{\mathrm{a}}$ & 12.40 & 59.35 & North Dakota State University \\
\hline Norm $^{a}$ & 13.32 & 62.22 & University of Minnesota \\
\hline Dalen & 14.12 & 65.54 & AgriPro \\
\hline Krona & 14.48 & 67.79 & AgriPro \\
\hline Wheaton ${ }^{\mathrm{a}}$ & 16.21 & 63.78 & University of Minnesota \\
\hline Minnpro & 16.22 & 57.10 & University of Minnesota \\
\hline Vance $^{\mathrm{a}}$ & 18.52 & 76.44 & University of Minnesota \\
\hline \multirow[t]{2}{*}{2398} & 31.66 & 84.21 & North Dakota Research Foundation \\
\hline & $(P=0.05)=3.42$ & $\operatorname{LSD}(P=0.05)=$ & \\
\hline
\end{tabular}

${ }^{a}$ Entry was also included in the 1995 trials (5 of the 13 bulked selections were included).

${ }^{\mathrm{b}}$ Minnesota selections of a larger set of Pioneer Hi-bred lines.

Table 2. Coefficients of determination $\left(r^{2}\right)$ for year-to-year comparisons of measurements of scab of wheat

\begin{tabular}{lcccc}
\hline Years & No. entries & Incidence $^{\text {a }}$ & Severity & Index \\
\hline $1992-1993$ & 97 & $0.57^{* \mathrm{~b}}$ & 0.42 & 0.58 \\
$1992-1993$ & 37 & 0.66 & 0.53 & 0.78 \\
$1992-1994$ & 37 & $0.69^{*}$ & 0.43 & 0.76 \\
$1993-1994$ & 37 & 0.64 & $0.80^{*}$ & 0.76 \\
\hline
\end{tabular}

a Incidence $=$ proportion of heads infected; severity $=$ proportion of spikelets infected on infected heads; index $=$ incidence $\times$ severity. Data transformed by arcsin $\sqrt{ }$ proportion.

${ }^{\mathrm{b}}$ Asterisk indicates a significantly higher $r^{2}$ value in comparisons of incidence versus severity for each pair of years. to establish similarities and differences among years or disease incidence, severity, or index measurements. We chose to use coefficients of determination, $r^{2}$, to describe both correlations and regressions because this statistic describes exactly how much influence one measure or year describes the other. While to many this may imply cause and effect, it is not intended this way for year-to-year comparisons, and it can easily be converted to correlation coefficient, $r$. Regression lines are shown in the figures and were the basis for slope comparisons. For statistical comparisons, arcsine transformations of the square root of proportions were used to normalize data, to minimize variance differences between measures being compared, and because this transformation is recommended for proportions (12). Transformed data were also used to obtain least significant differences.

Graphs were prepared using transformed data except in the case of 1992 and 1993 incidence versus severity, when untransformed values of the large set of entries were relatively evenly dispersed. The spreadsheet Excel (Microsoft, Seattle, WA) was used for transformations and arithmetic manipulation of data, and the graphing program Cricket Graph (Cricket Software, Malvern, PA) was used for regression and correlation analysis, curve fitting, and graphing.

\section{RESULTS}

Thirty-seven lines were tested the first 3 years (Table 1). These included moderately and highly susceptible named or numbered cultivars of hard red spring wheats from the northern Great Plains states, Sumai 3, a Chinese resistance source, and 13 genetically distinct Minnesota selections from the former breeding program of Pioneer Hi-bred International Company. The latter selections had moderately high to high levels of resistance derived from Chinese and other Asian sources. Nearly all lines with high resistance (arbitrarily set at mean disease index of less than $2 \%$ ) were derived from crosses with Chinese or other Asian sources of resistance. Cultivars not derived from Chinese sources that were moderately resistant included Butte 86, Sharp, and 2375. Marshall, reported to have fair to good resistance (15), was moderately susceptible.

Repeatability of disease measurements from year to year. Table 2 summarizes the fidelity of the two measures of resistance, incidence and severity, and their product, disease index, from 1 year to another for the first 3 pairs of years. In general, repeatability is very high, as expressed by the coefficient of determination, $r^{2}$, of each measure, with different years plotted on the x- and y-axes (Fig. 1). By inspection, and because $r^{2}$ values increased little when higher order fits were attempted, linear fits appeared adequate to describe relationships. The $P$ values asso- 
ciated with $r^{2}$ values are less than 0.0001 . Table 2 gives $r^{2}$ values for arcsine transformed data.

The disease index had higher year-toyear coefficients of determination than either incidence or severity, with $r^{2}$ values ranging from 0.76 to 0.78 for transformed disease indexes. Overall, lower coefficients of determination were obtained for incidence (mean transformed $r^{2}$ value of $0.663)$ and severity (0.587) than for disease index (0.767). Based on the range, severity coefficients of determination were more variable from year to year than incidence or disease index. High severity coefficients of determination were found in the 1993 to 1994 comparisons, even though these 2 years were the least correlated of the 3 pairs of year-to-year comparisons for incidence and disease index (Table 2).

A larger set of 97 cultivars or lines was examined in the 1992 and 1993 plots. This set included all lines in the smaller set, 39 additional Minnesota selections from the Pioneer lines not continued in 1994, additional breeding lines and cultivars from the Dakotas and Montana, and a few historically important cultivars such as Chris and Era. Coefficients of determination between these 2 years were considerably lower with this larger data set than they were for the small set, being 0.57 for incidence, 0.42 for severity, and 0.58 for disease index (all data arcsine transformed).

The 1995 season. This was intended to be a 3-year study. In 1995, however, 22 of the 37 lines (identified in Table 1) were planted in replicated rows in inoculated plots. These 22 lines cover the range of disease indexes measured in 1992 to 1994 in the set of 37 . For these 22 lines, correlations of 1995 incidence, severity, and index with the other 3 years were all nonsignificant. For example, 1995 index coefficients of determination were $0.02,0.002$, and 0.004 (untransformed), respectively, with 1992, 1993, and 1994. This lack of correlation contrasts sharply with the highly significant $r^{2}$ values found among all 3 pairs of years in 1992 to 1994. So, while the first 3 years ranked the cultivars in a similar manner, the fourth did not, indicating that the environment in 1995 greatly reduced the fidelity of scab resistance assessment.

The incidence-severity relationship. Figure 2 presents the scatter plots and regression lines for severity over incidence for each of the 3 years that the 37 lines were studied. The relationships are close all 3 years, with the lowest $r^{2}$ being in 1992. No lines could be identified that clearly possessed resistance due only to reduced incidence or reduced severity. The smaller set of 22 lines included in 1995 continued to show a high correlation between incidence and severity (Table 3). Figure 3 shows the relationship for the set of 97 lines included in 1992 and 1993. While the relationship between incidence and severity was close both years, the rela- tionship was linear only during 1992. Higher order polynomial relationships did not result in an increased $r^{2}$ value over that of a linear relationship for 1992, whereas a third-order equation, which fit the trend well in 1993 (by inspection), improved the relationship considerably (Table 3 ). Mean values for incidence and severity also show how the year interacted with the relationship. Mean incidence value in 1993 was $54.2 \%$, nearly twice that of 1992 . Yet mean severity dropped slightly from 1992 to 1993, going from 16.6 to 15.9 . As can be seen from the shape of the curve, in 1993 , the largest reduction in severity compared with 1992 was in the moderately resistant and moderately susceptible lines (from about 10 to $20 \%$ severity).

\section{DISCUSSION}

Ideally, plotting different years on the $\mathrm{x}$ and y-axes, all slopes might be expected to approximate 1.0. Only the comparison of 1992 with 1994 shows this. Because weather during 1993 was conducive to scab development, slopes for 1992 to 1993 and 1993 to 1994 were significantly higher and lower than 1.0, respectively, at alpha = 0.05 . This reflected the axis that 1993 data were assigned to in both cases.

During the first 3 years of our study, incidence and severity evaluations of germ plasm were consistent. However, 1995 evaluations of incidence and severity were unrelated to any of the previous 3 years. We believe that the repeatability of the first 3 years provided an accurate assessment of germ plasm and allowed effective selection for improved scab resistance. Problems were encountered in disease scoring in 1995 that were not present in 1992 to 1994. First, the wheat was about $50 \%$ lodged when assessed, due to high winds and rain that occurred just prior to anthesis of the earliest lines. This lodging was almost random in its distribution and affected replicate rows differently, possibly because neighboring rows consisted of different lines in each block. Within rows, the center of the row was commonly lodged, while the end plants remained standing. Lodged heads often had noticeably higher disease severity values than standing heads. Lodged plants could not be avoided and were included as they were encountered. Second, plant growth and development were accelerated in 1995 because of unusually warm weather. Readings were made 5 to 18 days earlier in 1995 than in the earlier years, as measured from the date of anthesis. It was more difficult, because of the speed of development of the lines and their large number, to time the readings as carefully as in other years. Accu-

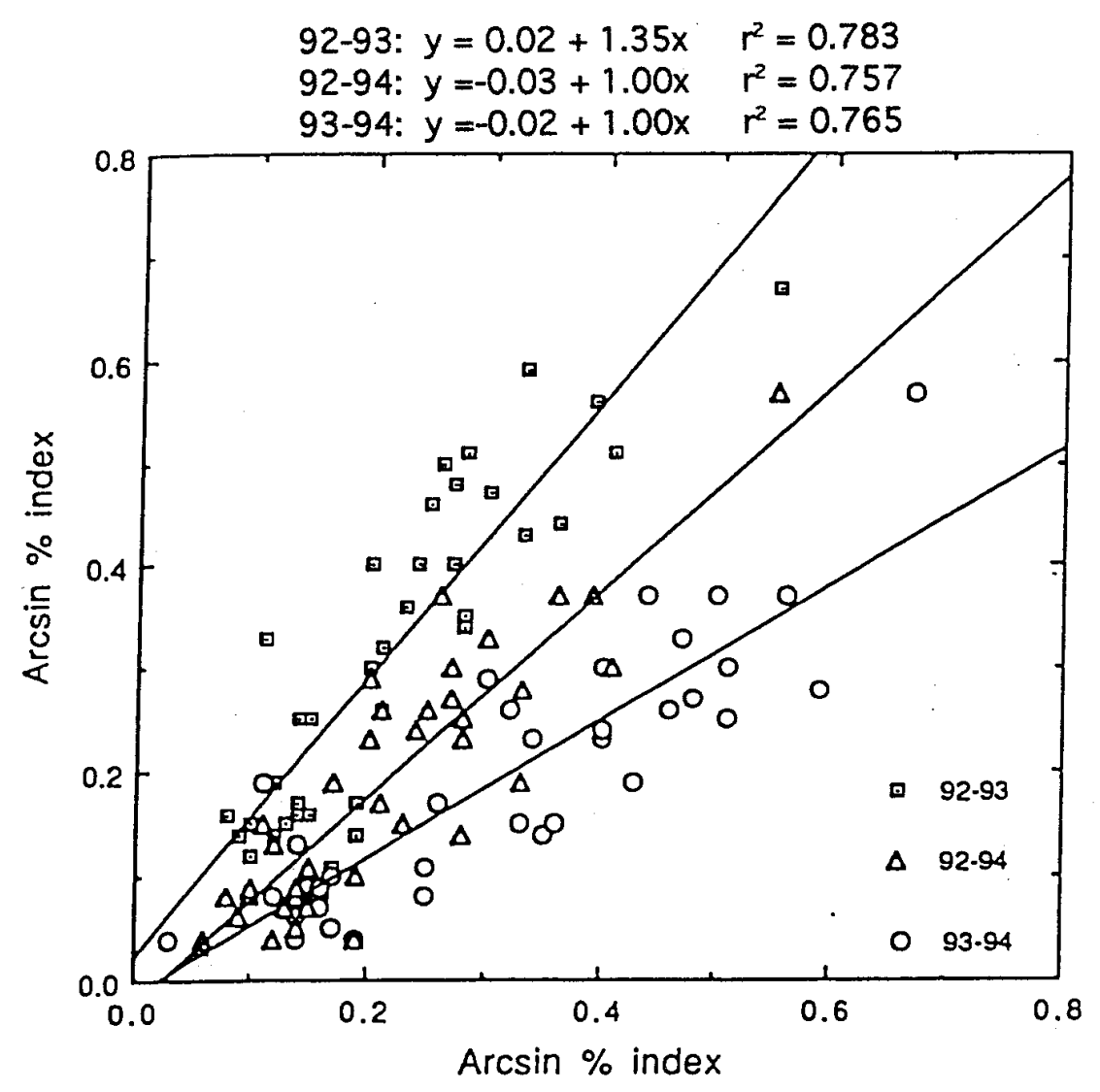

Fig. 1. Scatter plot of the relationships between years of the index measure of scab (ratio of diseased spikelets to total spikelets) in inoculated field plots for 37 spring wheat lines. Each point is the mean of three replicates, transformed to arcsine of the square root of the ratio. Linear regression lines and equations are also shown. 
racy of the evaluations was affected because severity increases daily under irrigation, and the optimum time for evaluation is when disease has progressed as far as it can before maturity of the spikelets prevents accurate scoring of disease. Because of the accelerated development of disease in 1995, at least six persons were employed as evaluators, compared with two in the other 3 years. This undoubtedly decreased accuracy of readings. The adverse effect of environment, which can cause genotype $\times$ environment interactions, may not be controllable, but their recognition can still permit accurate screening of lines for resistance in the field. In general, results from the 1995 field plot were not useful for breeding purposes.

Christensen et al. (4) in the early 1920s examined repeatability of scab assessment and the relationship between incidence and severity in inoculated plots containing adapted wheat germ plasm. In a series of comparisons of different locations, different years, or replicate "series" in the same year and location, coefficients of determination were low for either incidence or severity for the same series of lines. Their $r^{2}$ values for 41 to 71 lines of bread wheat ranged from 0 to 0.42 , with a mean of 0.16 . Incidence $r^{2}$ s were lower than those of severity. In five location or year comparisons where both measures were reported, incidence had a mean $r^{2}$ of 0.09 and severity a mean $r^{2}$ of 0.15 . They concluded that repeatability was generally poor. They gave little detail of methods used for inoculation, except that it involved repeated spraying of heads, beginning at emergence, with spore suspensions from many bulked cultures of $F$. graminearum. Hansen et al. (6) examined repeatability for pairs of years which included from 52 to 99 lines in each year-pair. Their results were similar to those of Christensen et al. (4). In 13 yearpairs, $r^{2}$ values for incidence (severity was not measured) averaged 0.21 and ranged from 0 to 0.45 . Low repeatability in the above studies might be due to a narrower range of resistance, because lines with high degrees of resistance were not present in their plots. A more recent study by Bekele et al. (3) used a set of seven wheat cultivars with low to moderate levels of resistance in uninoculated and inoculated plots at two Minnesota locations. Coefficients of determination between inoculated and uninoculated plots and between locations were generally low, with an average $r^{2}$

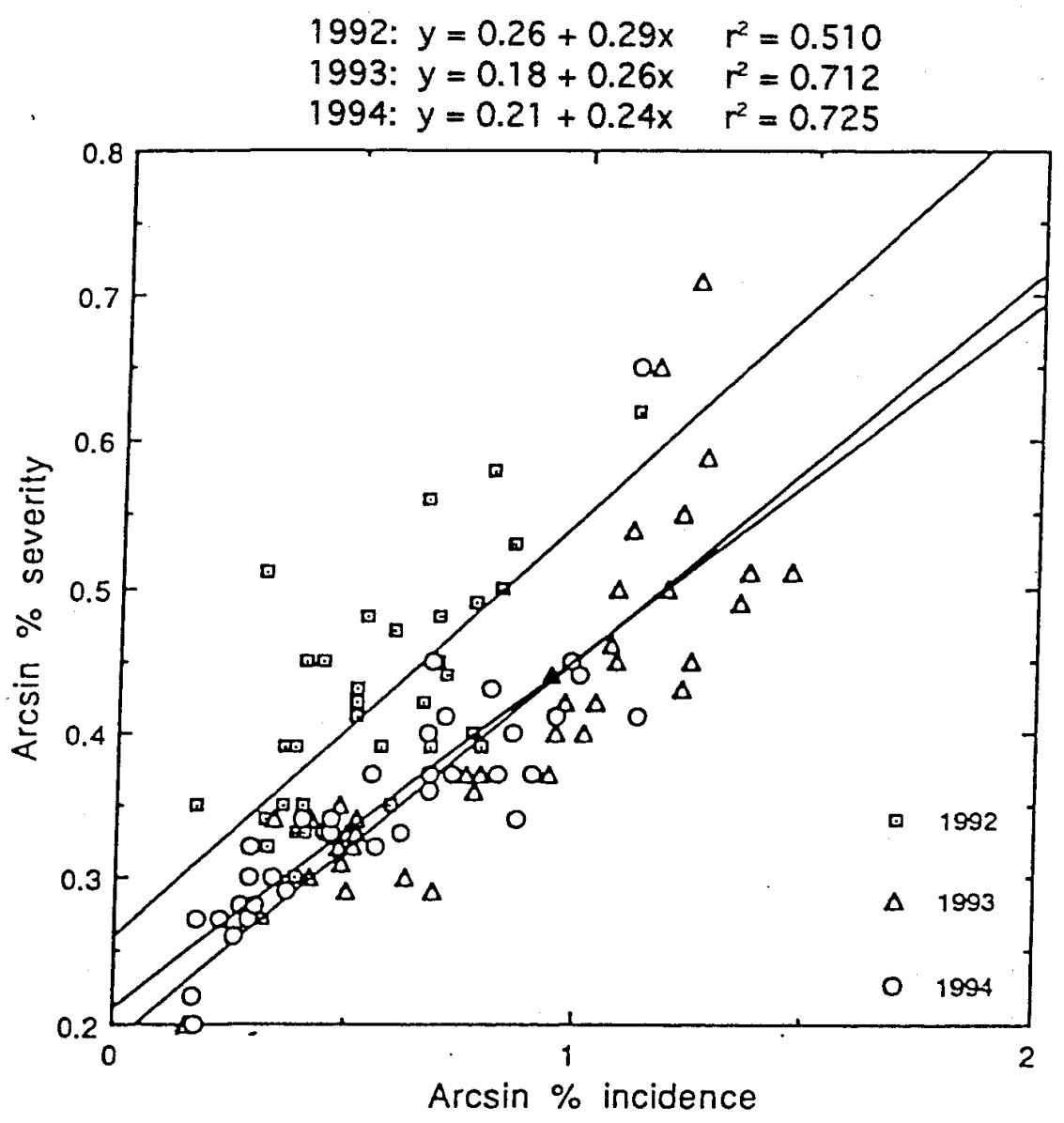

Fig. 2. Scatter plot of the relationships, shown separately for each of 3 years, between scab incidence and severity in inoculated field plots for 37 spring wheat lines. Each point is the mean of three replicates, transformed to arcsine of the square root of incidence and severity expressed as a proportion. Linear regression lines and equations are also shown. value of 0.32 for visual ratings of overall disease.

In contrast to the above rather low correlations, incidence and severity were highly correlated in the studies by Christensen et al. (4). They reported $r^{2}$ values that were no lower than 0.74 , and that averaged 0.80 in bread wheat plots containing from 41 to 154 lines. These values are similar to those reported in our studies. This is remarkable since there is virtually no overlap in the wheat cultivars or breeding lines of the two studies, although many of our cultivars would have parentage from some of the cultivars of Christensen et al.

If incidence and severity are distinct, as has been proposed for one wheat line (7), perhaps some lines possessing high levels of one or the other could be identified and used to combine high levels of the two kinds of resistance from separate sources. The high correlations we and others found between incidence and severity indicate that resistance components measured by percent incidence versus percent severity may not be distinct in the wheat lines that were examined. Conceivably, the same mechanisms could be responsible for resistance to both components of increase and spread of this disease. Those differences that we could see between incidence and severity can be explained by differences in timing of initial infection versus spread to other spikelets within infected heads. Conditions may be conducive for infection but not for spread in a given year. For example, the fact that incidence appeared to interact with year (Fig. 3), whereas severity did not, could simply be a matter of environmental conditions at the time of initial infection in 1993 that were more favorable than they were during the comparable period in 1992. Conditions at the time of spread within the heads were similar both years, resulting in little difference in mean severity.

Other sources of error may also call into question generalizations from our consistent results during 1992 to 1994 . Both the kind of inoculum used to generate the epidemic and the somewhat limited sample of wheat germ plasm may have affected our findings. For practical reasons, our inoculum consisted entirely of vegetatively propagated hyphal fragments and conidia of the pathogen. Stack (11) showed that asexual propagules and ascospores were

Table 3. Coefficients of determination $\left(r^{2}\right)$ for scab incidence-severity relationships of two sets of wheat lines in replicated, inoculated field plots ${ }^{\mathrm{a}}$

\begin{tabular}{ccc}
\hline Year & 37 entry set & 97 entry set $^{\mathbf{b}}$ \\
\hline 1992 & 0.51 & 0.58 \\
1993 & 0.71 & 0.62 \\
1994 & 0.72 & None \\
\hline
\end{tabular}

${ }^{a}$ All $r^{2}$ values are highly significant.

b This set includes all entries in the 37 entry set. Data transformed by arcsin $\sqrt{ }$ proportion. 
about equally effective in generating epidemics when applied to heads. More important than the kinds of propagules is the delivery as frequent, massive doses at concentrations that would be unlikely to occur with either ascospores or naturally produced conidia. Our study included a limited sample of resistant germ plasm primarily from China, including Sumai 3, Fan 1, Wuhan 3, Ning 7840, Ning 8331, and (non-Chinese) Frontana. Sumai 3 has been a widely used source of resistance because it has a good level of resistance that is more readily transferred than that from other sources (1). Sumai 3 is an important source of resistance in our lines, but many other sources are also represented. The sample of wheat germ plasm of Christensen et al. (4) showed an equally close relationship of incidence and severity, and supports the conclusion that the two components are not distinct. The number and diversity of lines in their studies were large but lacking in highly resistant representatives.

Bai and Shaner (1) report that studies in China $(2,13)$ indicate that spread of disease within the spike (severity) is a more stable trait to use to accurately characterize resistance than is incidence of spikes infected. The early studies of Christensen et al. (4) agree with this; repeatability of inci- dence measures between years, locations, or series was lower than repeatability of severity. In contrast, in the present studies, for 2 of the 3 pairs of years for the set of 37 lines, year-to-year repeatability was higher for incidence than it was for severity. Using a confidence interval about the correlation coefficient, $r$, we found that the $r$ value of incidence was significantly higher than that for severity for 1992 to 1994, but significantly lower for 1993 to 1994. The difference in $r$ values for 1992 to 1993 was not significant, although for the larger data set of 97 lines, $r$ for incidence was significantly higher than $r$ for severity. So, while a consistent picture was not obtained, incidence was certainly no less repeatable than severity, and in fact may have been slightly more repeatable. This incongruous pattern may reflect the fact that the environments of the individual years of those pairs in which severity showed lower $r$ values were different during the time of spread within the head, but not as different during initial infection. The disease index measure, which is the product of incidence and severity, was more repeatable than either of the components, possibly because environmental differences between years are being averaged, and a damping effect is the result. Because of the higher year-to-year fidelity of index

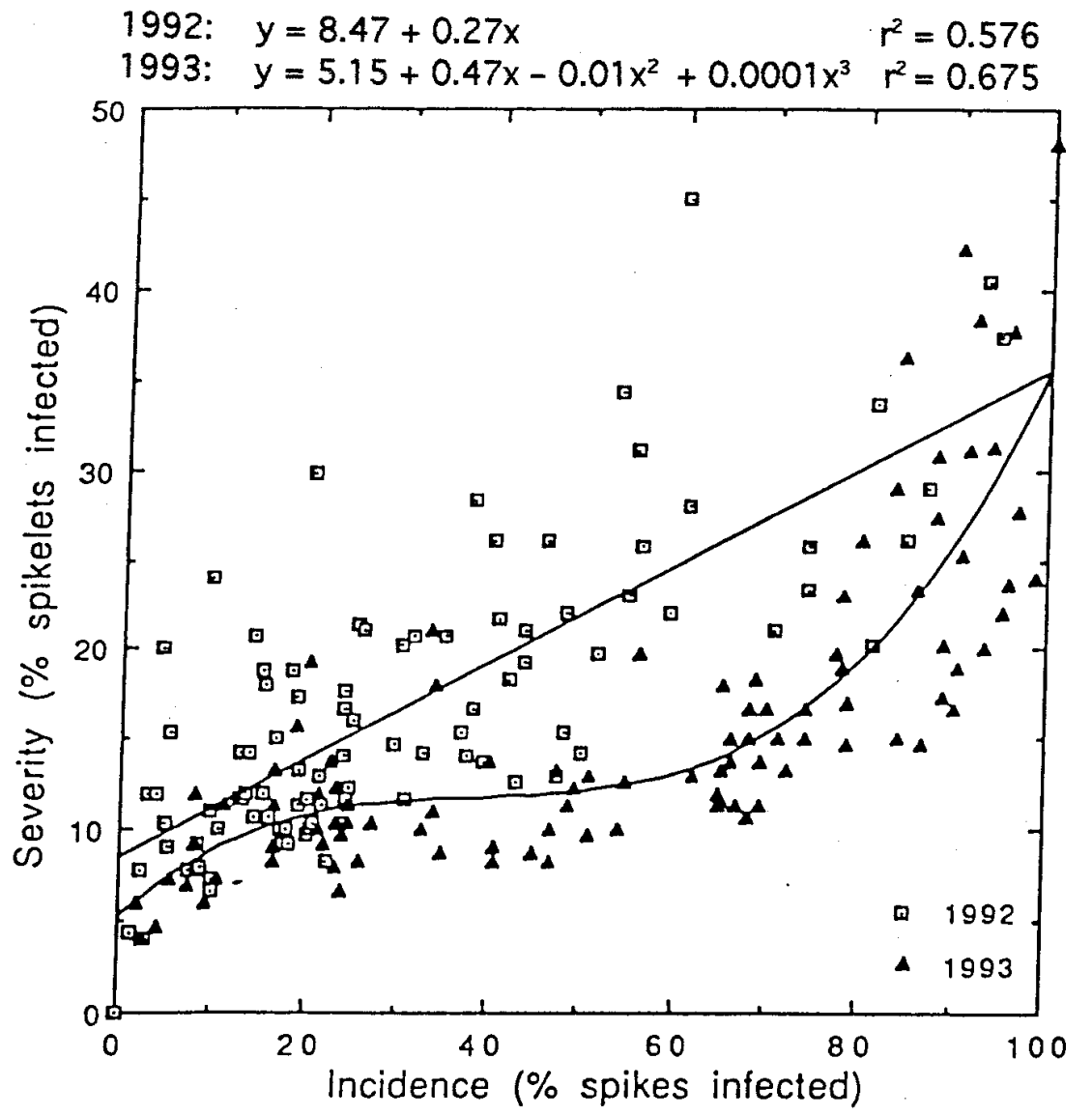

Fig. 3. Scatter plot of the relationships, shown separately for each of 2 years, between scab incidence and severity in inoculated field plots for 97 spring wheat lines. Each point is the mean of three replicates. Regression lines and equations are also shown.

compared with incidence or severity alone, we recommend that workers continue to assess both incidence and severity of scab in field nurseries.

Field evaluation of scab resistance in inoculated nurseries is still one of the most valuable methods of screening breeding germ plasm. Much of the progress in incorporating high levels of resistance into Chinese wheats took place in inoculated field nurseries (1). We determined that highly repeatable results can be obtained in field nurseries, so that increasing scab resistance by breeding is a reasonable goal, depending on the inheritance of resistance and its expression. Field nurseries are not used widely, however, because they are labor intensive, requiring much work in producing and disseminating inoculum, maintaining conducive disease conditions, and obtaining detailed data. Alternative methods have been reported. Seedling evaluation in the greenhouse, although potentially the easiest of all methods, has not proven to be useful, because correlations between seedling blight levels and scab resistance measured on adult plants are poor $(5,8,9,16)$. The correlations are probably too low to be of practical value generally, although seedling screening may prove useful to identify specific sources of resistance (5) or to eliminate very susceptible material (16). Greenhouse screening of adult plants using dipping or injection of heads or spikelets can provide accurate information on resistance to spread within the head (severity resistance) (1). The results from the present study suggest that severity resistance will usually be accompanied by resistance to initial infection of heads (incidence resistance), making these greenhouse methods more valuable.

\section{ACKNOWLEDGMENT}

We thank R. D. Wilcoxson and R. Dill-Macky for advice and assistance. Published as paper 991220092 of the contribution series of the Minnesota Agricultural Experiment Station based on research conducted under Project 22-76H, supported by the Minnesota Wheat Council and the Minnesota Crop Improvement Association.

\section{LITERATURE CITED}

1. Bai, G. H., and Shaner, G. 1994. Scab of wheat: Prospects for control. Plant Dis. 78:760-766.

2. Bai, G. H., Zhou, C. F., Qian, C. M., Xia, S. S., and Ge, Y. F. 1989. An analysis on combining ability of resistance to scab and other characters in eight wheat cultivars. Jiangsu Agric. Sci. Suppl. 1:79-83.

3. Bekele, G. T., Wilcoxson, R. D., Suganda, T. Busch, R. H., and Warnes, D. D. 1994. Comparison of methods for estimating head blight reactions of spring wheat cultivars infected with Fusarium graminearum. Int. J. Trop. Plant Dis. 12:89-100.

4. Christensen, J. J., Stakman, E. C., and Immer, F. R. 1929. Susceptibility of wheat varieties and hybrids to Fusarial head blight in Minnesota. Minn. Agric. Exp. Stn. Tech. Bull. 59.

5. Etebarian, H. R., and Wilcoxson, R. D. 1993. Seedling blight and head blight reactions of spring wheat cultivars to Fusarium culmorum. Plant Pathol. (Trends Agric. Sci.) 1:1-5. 
6. Hanson, E. W., Ausemus, E. R., and Stakman, E. C. 1950. Varietal resistance of spring wheats to Fusarial head blight. Phytopathology 40:902-914.

7. Li, Y. F., and Yu, Y. J. 1988. Monosomic analysis for scab index in wheat cultivar "WZHHS." J. Huazhong Agric. Univ. 7:327331.

8. Mesterhazy, A. 1978. Comparative analysis of artificial inoculation methods with Fusarium spp. on winter wheat varieties. Phytopathol. Z. 93:12-25.

9. Mesterhazy, A. 1987. Selection of head blight resistant wheats through improved seedling resistance. Plant Breed. 98:23-36.

10. Nelson, P. E., Toussoun, T. A., and Marasas, W. F. O. 1983. Fusarium Species: An Illustrated Manual for Identification. Pennsylvania State University, University Park.

11. Stack, R. W. 1989. A comparison of the inoculum potential of ascospores and conidia of Gibberella zeae. Can. J. Plant Pathol. 11:137142.

12. Steel, R. G. D., and Torrie, J. H. 1960. Principles and Procedures of Statistics. McGrawHill, New York.

13. Wang, Y., Yang, X., and Hsiao, C. 1982. The improvement of identification techniques of scab (Gibberella zeae Petch) resistance of wheat and the development of resistant sources. Sci. Agric. Sinica 5:67-77.

14. Wiese, M. V. 1987. Compendium of Wheat Diseases 2nd ed. American Phytopathological Society, St. Paul, MN.

15. Wilcoxson, R. D., Busch, R. H., and Ozmon, E. A. 1992. Fusarium head blight resistance in spring wheat cultivars. Plant Dis. 76:658-661.

16. Woodward, R. P., and Wilcoxson, R. D. 1991. Comparison of greenhouse seedling blight and field scab induced by Fusarium graminearum in spring wheats. (Abstr.) Phytopathology 81:1146. 